\title{
Cognitive coping and childhood anxiety disorders
}

\author{
Jeroen S. Legerstee $\cdot$ Nadia Garnefski $\cdot$ \\ Francine C. Jellesma $\cdot$ Frank C. Verhulst . \\ Elisabeth M. W. J. Utens
}

Received: 4 December 2008 / Accepted: 3 August 2009/Published online: 1 September 2009

(C) Springer-Verlag 2009

\begin{abstract}
To investigate differences in cognitive coping strategies between anxiety-disordered and non-anxious 911-year-old children. Additionally, differences in cognitive coping between specific anxiety disorders were examined. A clinical sample of 131 anxiety-disordered children and a general population sample of 452 non-anxious children were gathered. All children filled out the child version of the Cognitive Emotion Regulation Questionnaire (CERQ-k). Structured clinical interviews were used to assess childhood anxiety disorders. Results showed that anxiety-disordered children experience significantly more 'lifetime' negative life events than non-anxious children. Adjusted for the 'lifetime' experience of negative life events, anxiety-disordered children scored significantly higher on the strategies catastrophizing and rumination, and significantly lower on the strategies positive reappraisal and refocus on planning than non-anxious children. No significant differences in cognitive coping were found between children with specific anxiety disorders. Anxietydisordered children employ significantly more maladaptive and less adaptive cognitive coping strategies in response to
\end{abstract}

J. S. Legerstee · F. C. Verhulst · E. M. W. J. Utens ( $)$

Department of Child and Adolescent Psychiatry, Erasmus University Medical Centre Rotterdam/Sophia

Children's Hospital, Dr. Molewaterplein 60, PO Box 2060,

3000 CB Rotterdam, The Netherlands

e-mail: e.utens@erasmusmc.nl

N. Garnefski

Department of Clinical and Health Psychology,

Leiden University, Leiden, The Netherlands

F. C. Jellesma

Department of Educational Sciences, University of Amsterdam,

Amsterdam, The Netherlands negative life events than non-anxious children. The results suggest that cognitive coping is a valuable target for prevention and treatment of childhood anxiety problems.

Keywords Anxiety disorders · Cognitive coping · Cognitive emotion regulation - Children - Life events

\section{Introduction}

Anxiety disorders are the most prevalent psychiatric disorders in children [2,4]. Considering the high prevalence and persistence of childhood onset anxiety disorders and the association with considerable impairments in social and academic functioning $[15,16]$, efficient early prevention and intervention programs for this particular group of children are of great importance [4]. Such prevention and intervention programs could benefit from knowledge on the differential characteristics between clinically anxious and non-anxious children. Previous studies in adolescents and adults have shown that anxious and non-anxious individuals can be discriminated as to their use of specific cognitive coping strategies [9-11]. Cognitive coping, however, has never been examined in relation to childhood anxiety disorders before.

Cognitive coping can be defined as the cognitive way of managing the intake of emotionally arousing stimuli $[6,9$, 30]. Cognitive coping is distinguished from other partially overlapping constructs, such as emotion regulation and mood regulation, by its predominant focus on decreasing negative affect in response to stressful situations $[14,28]$. Cognitive coping is considered to act as a mediator and moderator of the association between stress and psychological well being $[6,7]$. Cognitive coping might be particularly important to examine in relation to childhood 
anxiety disorders, as anxiety-disordered children experience significantly more stressful situations (i.e., negative life events) than their non-anxious counterparts and perceive these situations as more threatening [1, 13, 19]. Maladaptive or excessive use of cognitive coping strategies might contribute to the development and persistence of anxiety disorders in children.

A recent study [17] showed that anxiety-disordered adolescents experience significantly more negative life events than non-anxious adolescents. In response to these life events, anxiety-disordered adolescents use emotionally adaptive (e.g., refocus on planning) as well as maladaptive (e.g., rumination, self-blame, catastrophizing and acceptance) cognitive coping strategies to a significantly higher extent than their non-anxious counterparts [17]. The largest proportion of variance was explained by the strategies rumination, self-blame, and catastrophizing. These three strategies have also been related to anxiety symptoms in adolescents and adults from the general population [9-11]. Apparently, anxiety-disordered adolescents not only experience more negative life events, but also tend to use more cognitive coping strategies, especially maladaptive, than non-anxious adolescents. With respect to specific anxiety disorders, adolescents with a generalized anxiety disorder appeared to make more use of the strategy rumination than adolescents with other specific anxiety disorders.

The results on cognitive coping in anxious adolescents and adults may not be simply generalized to children, because of large cognitive and emotional developmental differences. Childhood is a sensitive period in which children become progressively more aware of their internal emotional experiences [3,21], show a gradual maturation of their cognitive capacities [8], and begin to develop sophisticated cognitive strategies to regulate emotions [24, 29]. Children become progressively more able to regulate their emotions in response to stress in a cognitive way [20, 24]. Recently, a questionnaire (i.e., Cognitive Emotion Regulation Questionnaire-kids version; CERQ-k) was developed to assess cognitive coping strategies in 9-11-year-old children [12].

Garnefski et al. [12] examined the association between nine cognitive coping strategies, assessed by the CERQ-k, and fearfulness in a community sample of 717 children. A substantial percentage (i.e., 28\%) of the variance in fearfulness in children could be explained by the use of cognitive coping strategies. In accordance with previous studies in adolescents and adults, the strategies rumination, self-blame, and catastrophizing were positively related to fearfulness. The strategy positive reappraisal, however, had a strong negative association with fearfulness, suggesting a protective value. It is, however, unknown whether the results also apply to clinical anxiety problems in children.

The present study examined cognitive coping strategies in anxiety-disordered children, aged 9-11-years-old. A clinical sample of 131 anxiety-disordered children was included and diagnoses were obtained by structured clinical interviews. The main aim of the study was to compare anxiety-disordered and non-anxious children as regards to their use of cognitive coping strategies, taking into account the experience of negative life events. Additionally, the use of cognitive coping strategies was compared between different anxiety disorder subtypes. The outcomes of this study may provide more insight into the role of cognitive coping in childhood disorders as well as may give important clues for prevention and intervention.

Based on the original study on the CERQ- $k$ by Garnefski et al. [12], we hypothesized that anxiety-disordered children would use significantly more 'maladaptive' cognitive coping strategies (i.e., self-blame, catastrophizing and rumination) and less 'adaptive' cognitive coping strategies (i.e., positive reappraisal; positive refocusing) in response to negative life events than non-anxious children. With respect to cognitive coping in specific anxiety disorder subtypes, we hypothesized that children with a generalized anxiety disorder would make more use of the strategy rumination as compared to other anxiety disorder subtypes. We formulated this specific hypothesis because rumination is one of the key defining features of generalized anxiety disorder and as our previous study [17] in adolescents has shown that the strategy rumination is specifically related to generalized anxiety disorders.

\section{Methods}

Sample and procedure

Anxiety-disordered child sample

Eligible for participation were children (aged 9-11) consecutively referred between January 2005 and May 2008 to the Department of Child and Adolescent Psychiatry of the Erasmus Medical Centre-Sophia Children's Hospital. Before intake, 179 children were sent the Cognitive Emotion Regulation Questionnaire-kids version (CERQ-k) [12] to their home addresses. As a part of the routine intake procedure, all children and their parents were interviewed with the Anxiety Disorders Interview Schedule for Children (ADIS-C) [26]. In total, 131 children fulfilled the criteria of an ADIS-C anxiety diagnosis.

The anxiety-disordered child sample consisted of 53 girls and 78 boys with a mean age of 9 years and 11 months ( $\mathrm{SD}=9$ months). Table 1 shows the rate of pure (i.e., one single anxiety diagnosis without other comorbid anxiety disorders) and co-morbid anxiety disorders in the anxiety-disordered child sample. The most prevalent pure anxiety disorder was social phobia $(31 \%)$, whereas 
Table 1 Rate of pure and co-morbid anxiety disorder diagnoses in the anxiety-disordered child sample

\begin{tabular}{|c|c|c|}
\hline Anxiety disorder subtype & $N(131)$ & $(\%)$ \\
\hline \multicolumn{3}{|c|}{ Generalized anxiety disorder } \\
\hline Pure & 12 & $(9.2)$ \\
\hline Co-morbid & 46 & $(35.1)$ \\
\hline \multicolumn{3}{|l|}{ Social phobia } \\
\hline Pure & 16 & $(12.2)$ \\
\hline Co-morbid & 41 & $(31.3)$ \\
\hline \multicolumn{3}{|l|}{ Specific phobia } \\
\hline Pure & 13 & $(9.9)$ \\
\hline Co-morbid & 38 & (29) \\
\hline \multicolumn{3}{|l|}{ Separation anxiety disorder } \\
\hline Pure & 9 & $(6.9)$ \\
\hline Co-morbid & 24 & (18.3) \\
\hline \multicolumn{3}{|l|}{ Panic disorder } \\
\hline Pure & 0 & $(-)$ \\
\hline Co-morbid & 2 & $(1.5)$ \\
\hline \multicolumn{3}{|l|}{ Agoraphobia } \\
\hline Pure & 1 & $(0.8)$ \\
\hline Co-morbid & 3 & (2.3) \\
\hline \multicolumn{3}{|c|}{ Post-traumatic anxiety disorder } \\
\hline Pure & 3 & (2.3) \\
\hline Co-morbid & 1 & $(0.8)$ \\
\hline \multicolumn{3}{|c|}{ Obsessive-compulsive disorder } \\
\hline Pure & 4 & (3.1) \\
\hline Co-morbid & 10 & (7.6) \\
\hline
\end{tabular}

generalized anxiety disorder (35\%) was the most prevalent co-morbid anxiety disorder. Seven children (5\%) had a co-morbid depressive disorder and $10(7.6 \%)$ a co-morbid dysthymic disorder. Sixty-three children (48\%) had one anxiety disorder, 47 children (36\%) had two anxiety disorders, and 21 children (16\%) had more than two anxiety disorders.

\section{Non-anxious child sample}

The non-anxious child sample was derived from the general population sample upon which the CERQ-k questionnaire was validated. We refer to Garnefski et al. [12] for a full description of the sample. The general population sample was recruited from 11 primary public schools. The same standardized recruitment procedures were used at the 11 schools. Children were included if they were between 9 and 11-years-old. Written parental consent was obtained for all participating children. Children, who were absent at the day of data collection, could fill out the questionnaires at some other moment. Seven hundred seventeen children (394 boys and 323 girls) filled out the Cognitive Emotion Regulation Questionnaire-kids version (CERQ-k) [12] and the Dutch version of the Revised Fear Survey Schedule for Children (FSSC-R) [22, 23]. These questionnaires were completed during regular school hours, under supervision of a teacher, and two graduate psychology students. The children were guaranteed anonymity in relation to their parents, teachers, and fellow students.

To unambiguously define a non-anxious child sample, children were excluded from the general population sample when their score on the FSSC-R was higher than average according to the Dutch FSSC-R norm scores [23]. The final non-anxious child sample consisted of 452 children (173 girls and 279 boys), with a mean age of 9 years and 9 months ( $\mathrm{SD}=8$ months).

Instruments

\section{Cognitive coping strategies}

Cognitive coping strategies in children were assessed with the kids version of the Cognitive Emotion Regulation Questionnaire (CERQ-k) [12]. The CERQ-k is an adaptation of the original CERQ [9], which is suitable for adults and adolescents aged 12 years or older. The items of the original CERQ were simplified and shortened. The CERQ- $\mathrm{k}$ was constructed as a self-report questionnaire for 9-11year-old children and assesses what children think after the experience of negative life events. The CERQ-k consists of 36 items measuring nine different subscales. Each subscale consists of four items ranging from 1 [(almost) never] to 5 [(almost) always]. The higher the subscale score, the more the specific cognitive coping strategy is used.

The CERQ- $\mathrm{k}$ subscales are: refocus on planningthinking about what steps to take and how to handle negative events; rumination - thinking about the feelings and thoughts associated with negative events; putting into perspective - thoughts of playing down the seriousness of an event or emphasizing the relativity when comparing it to other events; catastrophizing - thoughts of explicitly emphasizing the terror of what you have experienced; positive refocusing - thinking about joyful and pleasant issues instead of thinking about an actual event; positive reappraisal - thoughts of creating a positive meaning to an event in terms of personal growth; acceptance: thoughts of accepting what you have experienced and resigning yourself to what has happened; self-blame-thoughts of putting the blame of what you have experienced on yourself; and other-blame - thoughts of putting the blame of what you have experienced on the environment or another person.

The psychometric properties of the CERQ-k have been proven to be good. Factorial validity and criterion-related validity of the CERQ-k are satisfactory [12]. All subscales have been shown to have high internal consistency ranging from 0.72 to 0.85 . 
Anxiety disorders

The ADIS-C $[25,26]$ was used in the clinical child sample to assess the following DSM-IV anxiety diagnoses: generalized anxiety disorder, social phobia, specific phobia, separation anxiety disorder, panic disorder, agoraphobia, post-traumatic stress disorder, and obsessive-compulsive disorder. Additionally, depressive disorder and dysthymic disorder were assessed.

The ADIS-C consists of a child and parent interview. If the minimal requirements for a DSM-IV diagnosis were met, the parent or the child was asked to indicate on a 9-point scale (i.e., 0-8) to what extent the symptoms interfered with the child's daily life. Subsequently, the interviewer gave an interference rating (Clinician Severity Rating, CSR), on the same 9-point scale, for the child and parent interview, separately. If the CSR was 4 or higher a diagnosis was assigned. Several researchers [18, 27] have shown that the inter-rater and test-retest reliability of the ADIS-C are good to excellent.

Experienced and trained post-doctoral clinicians administered the ADIS-C. Clinicians met several times to ensure that the procedures and decision-making were alike. The items of the ADIS-C and the CERQ did not overlap.

\section{Anxiety symptoms}

The Dutch version of the FSSC-R [22, 23] was used in the non clinical child sample to assess anxiety symptoms. Children were asked to rate each item on a three-point fear scale ("None," "Some," or "A Lot"). The FSSC-R consists of 80 items divided over five subscales, namely, fear of failure or criticism, fear of the unknown, fear of harm of small animals, fear of danger or death, and medical fear. By summing up the subscale scores, a total score can be obtained. Ollendick et al. [22] and Oosterlaan et al. [23] have shown that the subscales and total scale have good internal consistency (Cronbach's alphas ranging from 0.92 to 0.95 ) and high test-retest reliabilities (Pearson's $r$ ranging from 0.61 to 0.76 ).

\section{Negative life events}

The experience of negative events during the past year and longer than one year ago were assessed in anxiety-disordered and non-anxious children with a self-report checklist. Life events that were measured are: (1) divorce of parents, (2) long-lasting or severe medical illness of brother/sister, (3) long-lasting or severe medical illness of parent(s), (4) long-lasting or severe medical illness of self, (5) death of parent(s), (6) death of brother/sister, (7) death of grandparent(s) (8) having been victim of a severe accident, (9) having been bullied a lot.
Statistical analysis

A multivariate analysis of covariance (MANCOVA) was performed to examine differences in cognitive coping strategies between the anxiety-disordered and non-anxious child sample. The nine cognitive coping strategies were included as dependent variables and group (i.e., anxiety disordered vs. non-anxious children) as independent variable. Gender as well as the interaction between gender and group was also included as independent variables, as a previous study [10] has shown that gender is both related to anxiety problems as well as to the use of cognitive coping strategies. Background variables that significantly differed between anxiety-disordered and non-anxious child sample were entered as covariates in the analyses. When the MANCOVA yielded a significant main effect, subsequent univariate $F$ tests were performed.

A similar MANCOVA was performed, in which anxiety-disordered children with co-morbid affective disorders (i.e., depressive and dysthymic disorders) were excluded from the analysis. Previous research in the general population has shown that cognitive coping is not only related to anxiety symptoms, but also to depressive symptomatology. Therefore, this exclusion procedure was performed to rule out the possibility that the relatively high co-morbidity rate of affective disorders in the anxiety-disordered child sample would bias the results. Additionally, a MANCOVA was performed to examine differences in cognitive coping strategies between children with a pure anxiety disorder. Specifically, children with a pure generalized anxiety disorder, social phobia, specific phobia, and separation anxiety disorder were compared as regards cognitive coping strategies.

Effect sizes were expressed as the percentage of variance accounted for, and were considered small (1.0 to $<5.9 \%$ of variance), medium $(5.9$ to $<13.8 \%$ ), and large (13.8\% or more), according to Cohen's criteria [5].

\section{Ethics}

This study was approved by the Committee for Medical Ethics, Sophia Children's Hospital/Erasmus Medical Centre, Rotterdam.

\section{Results}

Preliminary analyses

The anxiety-disordered and non-anxious child samples did not significantly differ as to the distribution of gender. The anxiety-disordered child sample (mean age 9 years and 11 months) was significantly older $(t=3.61 ; P<0.001)$ 
than the non-anxious child sample (mean age 9 years and 9 months). The use of cognitive coping strategies did not differ between children that experienced negative life events in the past year versus children that experienced negative life events longer than 1 year ago. Therefore, the experience of life events during the past year and longer than 1 year age was collapsed into a broader category (i.e., 'lifetime experience of negative life events). Anxietydisordered children reported significantly more 'lifetime' negative life events $(t=15.83, P<0.001)$ than non-anxious children. As regards 'lifetime' negative life events, $47 \%$ of the non-anxious children did experience one or more negative life events in contrast to $92 \%$ of the anxietydisordered children. Of the anxiety-disordered children, $24 \%$ experienced one 'lifetime' negative life event, $36 \%$ experienced two 'lifetime' negative life events, and 32\% experienced more than two 'lifetime' negative life events. Of the non-anxious children, $38 \%$ experienced one 'lifetime' negative life event and 9\% experienced two 'lifetime' negative life events. The most common 'lifetime' experienced negative life event in anxiety-disordered children was being bullied a lot (61\%). In non-anxious children, the most common 'lifetime' experienced life event was the death of a grandparent $(26 \%)$. Parents were divorced in $24 \%$ of the anxiety-disordered children versus $3 \%$ of the non-anxious children.

As anxiety-disordered and non-anxious children differed in age and in the 'lifetime' experience of negative life events, both variables were entered as covariates in the subsequent statistical analyses.

\section{Differences in cognitive coping strategies}

between non-anxious and anxiety-disordered children

An overall difference in cognitive coping strategies was found between anxiety-disordered children and nonanxious children (Wilks' $\lambda=0.79 ; \quad F(9,346)=10.15$; $P<0.001$ ), explaining $20.9 \%$ of the variance. This is a large effect according to Cohen's criteria [5]. The covariates age $(F(9,346)=1.43 ; P=0.18)$ and 'lifetime' negative life events $(F(9,346)=1.11 ; P=0.35)$ did not explain a significant proportion of the variance in cognitive coping.

Subsequent univariate $F$ tests showed that anxietydisordered children scored significantly higher on the strategies self-blame, rumination, catastrophizing, and acceptance. On the other hand, anxiety-disordered children scored significantly lower on positive refocusing, positive reappraisal, and refocus on planning. Most of the variance was explained by rumination $(6 \%)$ and positive reappraisal $(5 \%)$. No significant interaction effect between gender and group was found (Wilks' $\lambda=0.98 ; F(9,346)=0.96$; $P=0.48)$.
Subsequent analyses, in which the 16 anxious children with co-morbid affective disorders were excluded, revealed an overall difference in cognitive coping between anxietydisordered and non-anxious children (Wilks' $\lambda=0.82$; $F(9,331)=8.25 ; \quad P<0.001)$, explaining $18.3 \%$ of the variance. No significant interaction effect between gender and group was found (Wilks' $\lambda=0.97 ; F(9,331)=0.98$; $P=0.46)$. Anxiety-disordered children scored significantly higher on the cognitive coping strategies catastrophizing and rumination, explaining 2 and $5 \%$ of the variance, respectively. However, anxiety-disordered children scored significantly lower on positive reappraisal and refocus on planning, explaining 4 and $2 \%$ of the variance, respectively. As in the previous analyses, most of the variance was explained by the strategies rumination and positive reappraisal (Table 2).

Differences in cognitive coping strategies between specific anxiety disorders

Statistical comparison of cognitive coping strategies between children with a pure generalized anxiety disorder, social phobia, specific phobia, and separation anxiety disorder revealed no significant differences (Wilks' $\lambda=0.54$; $F(27,99)=0.89 ; P=0.67)$. Additional comparison of the two most prevalent anxiety disorders in the child sample (i.e., social phobia and specific phobia) also revealed no significant difference (Table 3).

\section{Discussion}

Congruent to earlier findings $[1,19]$ the results of the present study showed that anxiety-disordered children experience significantly more negative life events compared to their non-anxious counterparts. Adjusted for the 'lifetime' experience of negative life events, anxiety-disordered, and non-anxious children differed in their use of cognitive coping strategies. These results were not modified by gender. Cognitive coping strategies explained a large amount of variance. With respect to specific cognitive coping strategies, anxiety-disordered children scored significantly higher on self-blame, rumination, catastrophizing, and acceptance and lower on positive reappraisal, positive refocusing, and refocus on planning. However, the results indicated that the differences found on the strategies self-blame, acceptance, and positive refocusing were attributable to affective disorder co-morbidity. Anxietydisordered and non-anxious children differed in particular on the strategies catastrophizing, rumination, positive reappraisal, and refocus on planning. Apparently, anxietydisordered children tend to think more about the feelings associated with negative life events and focus more on the 
Table 2 MANCOVA: comparison of cognitive coping strategies between anxiety-disordered and non-anxious children

\begin{tabular}{|c|c|c|c|c|c|c|c|c|}
\hline & \multicolumn{3}{|c|}{ Non-anxious } & \multicolumn{3}{|c|}{ Anxiety-disordered } & \multirow[t]{2}{*}{ Group* $(\%)$} & \multirow[t]{2}{*}{ Group $\times$ gender* $(\%)$} \\
\hline & $\begin{array}{l}\text { Girls } \\
(n=173) \\
M(S D)\end{array}$ & $\begin{array}{l}\text { Boys } \\
(n=279) \\
M(S D)\end{array}$ & $\begin{array}{l}\text { Total } \\
(n=452) \\
M(S D)\end{array}$ & $\begin{array}{l}\text { Girls } \\
(n=53) \\
M(S D)\end{array}$ & $\begin{array}{l}\text { Boys } \\
(n=78) \\
\text { M (SD) }\end{array}$ & $\begin{array}{l}\text { Total } \\
(n=131) \\
\text { M (SD) }\end{array}$ & & \\
\hline Self-blame & $8.1(3.2)$ & $7.2(2.9)$ & $7.5(3.1)$ & $9.2(4.4)$ & $8.5(4.2)$ & $8.8(4.3)$ & 1 & - \\
\hline Other-blame & $6.5(3.0)$ & $6.9(2.8)$ & $6.7(2.9)$ & $6.3(3.6)$ & $7.3(3.6)$ & $6.9(3.6)$ & - & - \\
\hline Rumination & $8.7(3.4)$ & $8.0(3.4)$ & $8.3(3.4)$ & $11.6(4.8)$ & $10.3(4.0)$ & $10.8(4.4)$ & 6 & - \\
\hline Catastrophizing & $7.6(3.2)$ & $7.5(3.2)$ & $7.5(3.2)$ & $9.3(3.9)$ & $9.7(4.3)$ & $9.5(4.1)$ & 2 & - \\
\hline Acceptance & $9.0(3.0)$ & $8.3(3.2)$ & $8.6(3.1)$ & $9.4(3.8)$ & $10.2(3.5)$ & $9.9(3.7)$ & 2 & - \\
\hline Putting into perspective & $10.9(3.7)$ & $10.8(4.1)$ & $10.8(3.9)$ & $9.3(3.7)$ & $9.8(3.4)$ & $9.6(3.5)$ & - & - \\
\hline Positive refocusing & $12.7(4.3)$ & $12.0(4.4)$ & $12.3(4.4)$ & $9.8(4.5)$ & $10.4(4.7)$ & $10.2(4.6)$ & 1 & - \\
\hline Positive reappraisal & $9.5(3.6)$ & $9.7(3.8)$ & $9.6(3.7)$ & $7.3(3.1)$ & $7.7(3.1)$ & $7.6(3.1)$ & 5 & - \\
\hline Refocus on planning & $10.7(3.7)$ & $10.2(4.1)$ & $10.4(4.0)$ & $9.1(4.6)$ & $8.8(3.5)$ & $8.9(3.9)$ & 2 & - \\
\hline
\end{tabular}

* Percentage of variance accounted for by significant group or by significant group x gender interaction effect; age and total number of life events were included as covariate; $P<0.05$

Table 3 MANOVA: comparison of cognitive coping strategies between children with a generalized anxiety disorder, social phobia, specific phobia, and separation anxiety disorder

\begin{tabular}{|c|c|c|c|c|c|}
\hline & $\begin{array}{l}\text { Generalized anxiety } \\
\text { disorder }(N=12) \\
\mathrm{M}(\mathrm{SD})\end{array}$ & $\begin{array}{l}\text { Social phobia } \\
(N=16) \\
\mathrm{M}(\mathrm{SD})\end{array}$ & $\begin{array}{l}\text { Specific phobia } \\
(N=13) \\
\mathrm{M}(\mathrm{SD})\end{array}$ & $\begin{array}{l}\text { Separation anxiety } \\
\text { disorder }(N=9) \\
\mathrm{M}(\mathrm{SD})\end{array}$ & Group* $(\%)$ \\
\hline Self-blame & $7.3(2.1)$ & $8.6(4.9)$ & $6.9(2.2)$ & $6.1(5.4)$ & - \\
\hline Other-blame & $7.0(2.9)$ & $8.4(4.6)$ & $5.4(2.7)$ & $5.4(2.1)$ & - \\
\hline Rumination & $10.2(3.9)$ & $8.9(3.6)$ & $8.9(3.3)$ & $8.5(2.4)$ & - \\
\hline Catastrophizing & $7.7(3.0)$ & $8.6(3.6)$ & $6.8(3.0)$ & $6.0(1.2)$ & - \\
\hline Acceptance & $8.8(3.4)$ & $10.4(2.6)$ & $9.6(4.4)$ & $9.4(3.4)$ & - \\
\hline Putting into perspective & $9.4(3.1)$ & $8.6(3.1)$ & $10.3(2.9)$ & $10.6(2.8)$ & - \\
\hline Positive refocusing & $10.9(5.5)$ & $10.5(4.40$ & $11.1(3.6)$ & $14.8(3.5)$ & - \\
\hline Positive reappraisal & $6.8(2.0)$ & $7.4(2.9)$ & $6.5(2.8)$ & $7.4(1.9)$ & - \\
\hline Refocus on planning & $8.8(4.1)$ & $9.0(4.8)$ & $8.0(5.1)$ & $9.4(3.3)$ & - \\
\hline
\end{tabular}

* Percentage of variance accounted for by significant group effect; $P<0.05$

negative aspects of what they have experienced compared to non-anxious children. Additionally, anxiety-disordered children use less thoughts of creating a positive meaning to an event in terms of personal growth, and think less about what steps to take and how to handle negative events. The strategies rumination and positive reappraisal accounted for most of the difference between anxiety-disordered and non-anxious children. The present results fit with the results of a recent study [12] in children from the general population. Garnefski et al. [12] also found that catastrophizing and rumination had a strong positive relation, and positive reappraisal a strong negative relation with fearfulness. These strategies, particularly catastrophizing and rumination, have also been related to anxiety symptoms in adolescents and adults from the general population [9-11].
The present results are noteworthy in comparison with our recent study in anxiety-disordered adolescents [17]. Anxiety-disordered adolescents appeared to score significantly higher than non-anxious adolescents on strategies that have been suggested to be maladaptive (e.g., rumination, self-blame, catastrophizing, and acceptance) as well as on strategies that are considered to be adaptive (e.g., refocus on planning) for psychological functioning. Contrary to clinically anxious children, anxiety-disordered adolescents did not score significantly lower on adaptive strategies. It seems that anxiety-disordered adolescents employ more cognitive efforts, both adaptive and maladaptive, to cope with negative life events, whereas anxiety-disordered children show a more distinguished pattern of cognitive coping strategies. Anxiety-disordered children 
specifically use more maladaptive and less adaptive cognitive coping strategies in response to negative life events compared to non-anxious children.

Childhood is considered an important period in which coping develops rapidly [28]. The development of a maladaptive coping repertoire in childhood might have negative consequences for the ability of a person the cope with negative life events later in life. It is of utmost importance to intervene when a child uses maladaptive and less adaptive cognitive coping strategies. The present study's results suggest that treatment programs for anxiety-disordered children should specifically focus on reducing maladaptive cognitive coping strategies as well as enhancing the use of adaptive cognitive coping strategies. Furthermore, the administration of the CERQ-k before the start of treatment might give clinical therapists the unique opportunity to deliver an individually tailored treatment program, based on a child's specific use of maladaptive and adaptive cognitive coping strategies. The CERQ-k might also be a valuable tool in monitoring changes in cognitive coping strategies over the course of treatment.

With respect to specific anxiety diagnoses, children with a generalized anxiety disorder, social phobia, separation anxiety disorder, and specific phobia did not differ in their cognitive coping strategies. Based on a recent study in adolescents with a generalized anxiety disorder, it was expected that children with a generalized anxiety disorder would score significantly higher on the strategy rumination. However, only a non-significant trend was found suggesting that children with a generalized anxiety disorder score higher on rumination than children with other specific anxiety diagnoses. An important limitation of the comparison of children with specific anxiety diagnoses was that the number of pure anxiety diagnoses (i.e., without affective disorder co-morbidity) was fairly small, which diminished statistical power. Statistical power analyses indicated that only large effect sizes could be detected with the number of pure anxiety diagnoses included in this comparison. Furthermore, the small number of pure anxiety diagnoses prevented us to examine cognitive coping strategies in other anxiety diagnoses, such as panic disorders, agoraphobia, obsessive-compulsive disorders, and post-traumatic anxiety disorders.

Several limitations of the present study are noteworthy to mention. It is important to acknowledge that due to the cross-sectional nature of this study, no conclusions can be drawn regarding the direction of influence. It is possible that using specific maladaptive cognitive coping strategies may cause anxiety disorders. On the other hand, cognitive coping may be an epiphenomenon of anxiety disorders. Another limitation was that the assessment of cognitive coping and the experience of life events were based on a self-report measure, which may have caused some bias. It cannot be excluded that anxiety-disordered children exhibit a different response style than non-anxious children, which may have influenced the results. Therefore, future studies should acknowledge differences in response styles by including multiple informants. Additionally, different measures were used in the anxiety-disordered and nonanxious child sample to select anxious children. To what extent this difference in measurements has influenced our findings is unknown. However, as we used a strict criterion (i.e., lower than the average according to the norms) to select non-anxious children from the general population sample, we assume that the risk of selection bias is small. Strength of the study was that anxiety diagnoses in the patient sample were obtained by a structured clinical interview in both parents and children, and that the items of the CERQ-k did not overlap with the ADIS-C.

To summarize, this study indicated that anxiety-disordered children employ significantly more maladaptive cognitive coping strategies, and less adaptive cognitive coping strategies, in response to negative life events as compared to non-anxious children. Gender does not modify the association between cognitive coping and childhood anxiety disorders. Children with specific anxiety diagnoses seem not to differ in their cognitive coping strategies. As anxiety-disordered children experience significantly more negative life events and tend to cognitively cope with these events in a relatively maladaptive way, future prevention and psychotherapeutic intervention studies on childhood anxiety may incorporate strategies to assess and address cognitive coping.

\section{References}

1. Allen JL, Rapee RM, Sandberg S (2008) Severe life events and chronic adversities as antecedents to anxiety in children: a matched control study. J Abnorm Child Psychol 36:1047-1056

2. Alyahri A, Goodman R (2008) The prevalence of DSM-IV psychiatric disorders among 7-10 year old Yemeni schoolchildren. Soc Psychiatry Psychiatr Epidemiol 43:224-230

3. Calkins SD, Fox NA (2002) Self-regulatory processes in early personality development: a multilevel approach to the study of childhood social withdrawal and aggression. Dev Psychopathol 14:477-498

4. Cartwright-Hatton S, McNicol K, Doubleday E (2006) Anxiety in a neglected population: prevalence of anxiety disorders in preadolescent children. Clin Psychol Rev 26:817-833

5. Cohen J (1988) Statistical power analysis for the behavioral sciences, 2nd edn. Erlbaum, Hillsdale, NJ

6. Compas BE, Connor-Smith JK, Saltzman H, Thomsen AH, Wadsworth ME (2001) Coping with stress during childhood and adolescence: problems, progress, and potential in theory and research. Psychol Bull 127:87-127

7. Connor-Smith JK, Compas BE (2002) Vulnerability to social stress: coping as a mediator or moderator of sociotropy and symptoms of anxiety and depression. Cogn Ther Res 26:39-55 
8. Flavell JH (2000) Development of children's knowledge about the mental world. Int J Behav Dev 24:15-23

9. Garnefski N, Kraaij V, Spinhoven P (2001) Negative life events, cognitive emotion regulation and emotional problems. Pers Individ Dif 30:1311-1327

10. Garnefski N, Kraaij V, van Etten M (2005) Specificity of relations between adolescents' cognitive emotion regulation strategies and Internalizing and Externalizing psychopathology. J Adolesc 28:619-631

11. Garnefski N, Legerstee J, Kraaij VV, Van Den Kommer T, Teerds J (2002) Cognitive coping strategies and symptoms of depression and anxiety: a comparison between adolescents and adults. J Adolesc 25:603-611

12. Garnefski N, Rieffe C, Jellesma F, Terwogt MM, Kraaij V (2007) Cognitive emotion regulation strategies and emotional problems in 9-11-year-old children: the development of an instrument. Eur Child Adolesc Psychiatry 16:1-9

13. Gothelf D, Aharonovsky O, Horesh N, Carty T, Apter A (2004) Life events and personality factors in children and adolescents with obsessive-compulsive disorder and other anxiety disorders. Compr Psychiatry 45:192-198

14. Gross JJ (2007) Handbook of emotion regulation. The Guilford Press, New York

15. Ialongo N, Edelsohn G, Werthamer-Larsson L, Crockett L, Kellam S (1995) The significance of self-reported anxious symptoms in first grade children: prediction to anxious symptoms and adaptive functioning in fifth grade. J Child Psychol Psychiatry 36:427-437

16. Langley AK, Bergman RL, McCracken J, Piacentini JC (2004) Impairment in childhood anxiety disorders: preliminary examination of the child anxiety impact scale-parent version. J Child Adolesc Psychopharmacol 14:105-114

17. Legerstee JS, Garnefski N, Verhulst FC, Utens EMWJ (in revision) Cognitive coping in anxiety-disordered adolescents. $\mathrm{J}$ Adolesc

18. Lyneham HJ, Abbott MJ, Rapee RM (2007) Interrater reliability of the anxiety disorders interview schedule for DSM-IV: child and parent version. J Am Acad Child Adolesc Psychiatry 46:731-736
19. Marteinsdottir I, Svensson A, Svedberg M, Anderberg UM, von Knorring L (2007) The role of life events in social phobia. Nord J Psychiatry 61:207-212

20. Muris P, Mayer B, Vermeulen L, Hiemstra H (2007) Theory-ofmind, cognitive development, and children's interpretation of anxiety-related physical symptoms. Behav Res Ther 45:21212132

21. Muris P, Meesters C, Eijkelenboom A, Vincken M (2004) The self-report version of the strengths and difficulties questionnaire: its psychometric properties in 8- to 13-year-old non-clinical children. Br J Clin Psychol 43:437-448

22. Ollendick TH (1983) Reliability and validity of the revised fear surgery schedule for children (FSSC-R). Behav Res Ther 21:685692

23. Oosterlaan J, Prins PJM, Sergeant J (1995) Vragenlijst voor angst bij kinderen (VAK): Nederlands bewerking van de revised Fear Survey Schedule for Children [Questionnaire for fear in children: Dutch adaptation of the revised Fear Survey Schedule for Children]. Gedragstherapie 28:297-300

24. Saarni C (1999) The development of emotional competence. Guilford Press, New York

25. Siebelink BM, Treffers PDA (2001) Anxiety disorder interview schedule for DSM-IV-child version/Dutch translation. SWETS Test Publishers, Lisse, The Netherlands

26. Silverman WK, Albano AM (1996) Anxiety disorders interview schedule for DSM-IV child version. The Psychological Corporation, San Antonio

27. Silverman WK, Saavedra LM, Pina AA (2001) Test-retest reliability of anxiety symptoms and diagnoses with the anxiety disorders interview schedule for DSM-IV: child and parent versions. J Am Acad Child Adolesc Psychiatry 40:937-944

28. Skinner EA, Zimmer-Gembeck MJ (2007) The development of coping. Annu Rev Psychol 58:119-144

29. Stegge H, Meerum Terwogt M, Reijntjes A, Van Tijen N (2004) Children's conception of the emotion process: consequences for emotion regulation. In: Nyklicek I, Temoshok L, Vingerhoets A (eds) Emotional expression and health. Routledge, Hove

30. Thompson RA (1991) Emotion regulation and emotional development. Educ Psychol Rev 3:269-307 